\section{A discovery and new puzzles}

\section{Washington}

INVESTIGATORS searching for the cause of the spherical aberration that afflicts the Hubble Space Telescope (HST) have pinned the blame on what had always been the prime suspect. Measurements made last week revealed that one of the elements of the reflective null corrector, an optical device used to assess the shape of the primary mirror during polishing, was misplaced by a millimeter. But this discovery still does not dispel the mystery of the HST: so gross a flaw in the null corrector is as baffling as the error in the primary mirror itself. And to add to the frustration, it has been found that a test of the primary mirror conducted with a second null corrector clearly showed the presence of aberration - but the evidence was discounted because the second corrector was thought to be of lower quality.

Null correctors are needed in the manufacture of astronomical mirrors because of the departure of the mirror profiles from spherical form. An exactly spherical surface has the property that light emitted from a point source at its centre of curvature will be reflected back along radii to the same spot; a failure to achieve focus in this way indicates that the mirror is not perfectly spherical. To test the shape of a hyperboloidal mirror such as HST's primary, a variation on this technique is used. A null corrector is interposed between the mirror and a light source in such a way that light returns to a single focus; the corrector compensates for the asphericity of the mirror. But if the null corrector is inaccurately designed or made, or if it is put in the wrong place with respect to the mirror surface, the mirror's surface will be faulty.

In the manufacture of the HST's primary, two null correctors were used at different stages. The supposedly less accurate one, a 'refractive' null corrector whose optical elements were lenses, was used to check that the shape of the mirror was approximately correct before final polishing began. The reflective null corrector, made of small mirrors rather than lenses, was then used in conjunction with a computer-controlled polishing apparatus to bring the mirror into its final form. Although both null correctors were made to high precision, optical engineers at Perkin-Elmer, the company that made the mirror, believed that reflecting rather than refracting optical elements should be more accurate, and thus that tests conducted with the reflective corrector were more trustworthy.

According to the announcement last week from the National Aeronautics and Space Administration (NASA), the design of the reflective null corrector was as it should have been, but somehow it was put together wrong. Roger Angel, a mirrorbuilder at the University of Arizona and a member of the HST board of investigation, says that there was no obvious carelessness at Perkin-Elmer: spacing rods between the components of the null corrector were made and verified to micron accuracy - but they somehow turned out to be off by a millimetre. Angel suggests that although an enormous amount of care was invested in following predetermined manufacturing and testing procedures for all parts of the HST's optical system, "common sense was lost". He points out that the error in the null corrector could have been detected, had anyone thought to try, with a standard plastic ruler.

An equally alarming discovery by the HST investigators was that the refractive null corrector had been used one last time to measure the overall radius of curvature of the mirror after polishing and figuring had been completed. The results of this test, found amnng the documents impounded by the board of investigation

\section{Washington}

DESPITE accidentally losing their $\$ 250,000$ camera platform more than a mile underwater, deep-sea researchers in Oregon were putting on a brave (if red) face last week. The chance arrival of a deep-diving submarine may mean the rescue of the expensive package, and the resumption of their exploration of volcanic fissures on the ocean floor.

The platform, containing a special camera and a series of related mapping instruments, now sits on the ocean floor 200

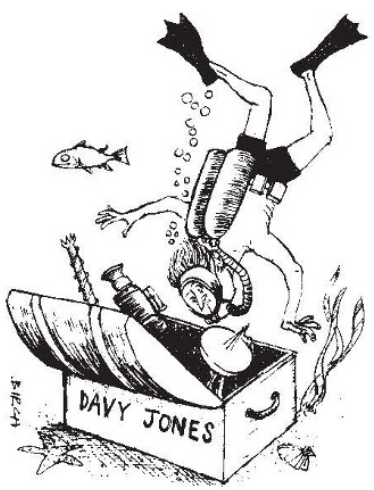

miles off the coast of Oregon, thanks to a defective cable that parted during a routine mapping process. Robert Embley, a geophysicist at the National Oceanic and Atmospheric Administration who is chief scientist for the project, says that his team thinks they have found the platform. They dropped a network of beacons and a special mooring platform (which consists of a (see Nature 346, 207; 1990), clearly revealed the spherical aberration. Apparently, however, confidence in the reflective null corrector was so great that this finding did not set off any alarm bells.

While Angel and his colleagues continue their work, trying now to discover how a millimetre error in the null corrector occurred and went undetected, other optical experts, including Marjorie Meinel of the Jet Propulsion Laboratory in California, are advising NASA on how best to correct the HST's aberration.

Meinel says that although knowledge of the flawed null corrector will help to pin down the exact form of the aberration and thus to design a corrective lens for the Wide Field and Planetary Camera (see Nature 346, 3; 1990), the most accurate measure of the aberration will come from direct imaging tests still being conducted by NASA. One possibility, according to Meinel, is to design corrective elements for the camera and other instruments which include some degree of adjustability, so that final precise error compensation can be done on board HST.

David Lindley

\title{
Full fathom five my camera lies
}

heavy weight connected to a number of glass floats and a very strong cable) in the area, to aid in the rescue.

Alvin, Woods Hole Oceanographic Institution's deep-diving submersible, will attempt an unusual rescue mission of the package this week. During the dive, its three-person crew will attempt to find the instrument by searching among the beacons. If they find it, they will try to attach it to the mooring platform and then release the weight. The floats should bring the cable to the surface, where a ship-borne winch can raise the entire 5,000-pound package.

Alvin's rescue attempt will be its second in a week. Last weekend, it was going to try to recover a side-scan radar instrument that a private company lost two months ago. About ten such scientific, mapping or deep-ocean drilling instruments are lost each year, says Barrie Walden, manager of submersible engineering and operations at Woods Hole. But Alvin, America's chief non-military submersible, is rarely available to attempt a rescue; it is called on only about once every two years. Alvin happens to have been conducting biological and geochemical observations of the same volcanic plume area where the two instruments were lost. "Most of the time when something like this happens, you're out of luck", says Embley.

That Alvin happens to have been scheduled for the area anyway, he says with some understatement, is a "fortuitous circumstance." ChristopherAnderson 